\title{
EDUCACIÓN Y SOCIEDAD DEL CONOCIMIENTO Y DE LA INFORMACIÓN
}

Juan Carlos Tedesco*

Quisiera comenzar esta conferencia excusándome por no referir específicamente al problema de la educación secundaría. Otros conferencistas lo harán con mucha más pertinencia de la que yo pueda alcanzar. He optado, en cambio, por presentarles un análisis más global de los procesos de cambio social que tienen lugar actualmente en nuestras sociedades $\mathrm{y}$, a partir de ese análisis, identificar algunos de los principales problemas y desafíos de la educación en general, que pueden servir de marco para ubicar las discusiones específicas sobre la enseñanza secundaria.

Ya se ha dicho repetidamente que el fin de siglo y la entrada en el nuevo milenio están asociados a un profundo proceso de transformación social. No estamos viviendo una de las periódicas crisis coyunturales del modelo capitalista de desarrollo, sino la aparición de nuevas formas de organización social, económica y política. La crisis actual, desde este punto de vista, es una crisis estructural, cuya principal característica es la simultaneidad de las dificultades de funcionamiento en las instituciones responsables de la cohesión social (la crisis del Estado-Providencia), en las relaciones entre economía y sociedad (la crisis del trabajo) y en los modos de constitución de las identidades individuales y colectivas (crisis del sujeto).

Durante algunos años, esta nueva configuración social fue descripta como postalguna dimensión de la sociedad: post-capitalista para Peter Drucker, post-industrial para Touraine, post-moderna para un amplio conjunto de intelectuales. Más recientemente, sin embargo, comenzó a difundirse y aceptarse una visión de esta nueva configuración social basada en la idea de que el rasgo central de la nueva organización social consiste en que el conocimiento y la información estarían reemplazando a los recursos naturales, a la fuerza y/o al dinero, como variables clave de la generación y distribución del poder en la sociedad.

Los primeros análisis acerca del papel del conocimiento y de la información como variables centrales del poder fueron significativamente optimistas acerca de sus potencialidades democratizadoras. Alvin Toffler fue, sin duda alguna, el representante más importante de esta corriente. Sus análisis se basaban en el carácter esencialmente democrático que tienen tanto la producción como la distribución de los conocimientos y las informaciones. Según Toffler, el conocimiento es infinitamente ampliable. Su uso no lo desgasta sino que, al contrario, puede producir aún más conocimiento. La producción de conocimientos requiere, además, un ambiente de creatividad y de libertad opuesto a toda tentativa autoritaria o burocrática de control del poder. Desde este punto de vista, la utilización intensiva de conocimientos produce la disolución de las formas burocráticas de gestión, porque obliga a renovar permanentemente las líneas de decisión en función de la acumulación y el intercambio de conocimientos. Por último, la distribución de

\footnotetext{
"Director de la Sede Regional del Instituto Internacional de Planeamiento de la Educación (IIPE). Buenos Aires, Argentina.
} 
conocimientos es mucho más democrática que la distribución de cualquier otro factor tradicional de poder, ya que - dice Toffler — "el débil y el pobre pueden adquirirlo".

Pero la evolución de la sociedad y particularmente de aquéllas que utilizan las nuevas tecnologías de producción basadas en el uso intensivo de conocimientos e información, mostró rápidamente que este optimismo era, por lo menos, ingenuo. Hoy disponemos ya de visiones más realistas y complejas acerca de los efectos sociales de los nuevos patrones de organización social y económica basados en el conocimiento y la información. La hipótesis más general sobre la cual se apoyan estas revisiones, consiste en sostener que una sociedad basada en el uso intensivo de conocimientos produce simultáneamente fenómenos de más igualdad y de más desigualdad, de mayor homogeneidad y de mayor diferenciación.

Permítanme comenzar por el aumento de la desigualdad. No pretendo aburrirlos con datos estadísticos, sino simplemente recordar aquí que uno de los fenómenos más importantes que se registra a través de numerosos indicadores es el significativo aumento de la desigualdad social en los últimos años y que este aumento es mayor en aquellos lugares donde tienen vigencia importantes procesos de transformación productiva y tecnológica. Los datos sobre la evolución de la distribución del ingreso en el mundo en las últimas días, por ejemplo, permiten apreciar que, en general, mientras los altos niveles de concentración de la riqueza se mantienen en los países en desarrollo, los países desarrollados, en cambio, están atravesando por un significativo proceso de concentración que los acerca a los países en desarrollo.

Todos sabemos que estos procesos son muy complejos y que no es posible atribuir el aumento de la desigualdad a un solo factor. Sin embargo, también se admite cada vez más que uno de los factores fundamentales asociado al aumento de la desigualdad es la transformación en la organización del trabajo. En pocas palabras, este fenómeno podría ser descripto diciendo que la incorporación de nuevas tecnologías al proceso productivo está asociada a la eliminación de numerosos puestos de trabajo. La mayor parte de los nuevos puestos de trabajo no se crean en los sectores tecnológicamente más avanzados, sino en los servicios, donde el costo del trabajo representa una proporción importante del precio del producto.

Esta diferencia en el ritmo de creación de puestos de trabajo está asociada a diferencias en los salarios, ya que mientras los sectores de alta productividad pueden tener políticas salariales generosas, los sectores de servicios, donde el vínculo entre salarios y empleo es muy alto, están obligados a aumentar muy moderadamente los salarios si quieren que crezca el empleo. Esta dinámica, donde el empleo disminuye en los sectores que pueden pagar buenos salarios y aumenta en aquéllos que pagan salarios modestos, explica las razones por las cuales la recomposición del empleo en función de la evolución tecnológica aumenta la desigualdad.

Pero las transformaciones en la organización del trabajo no sólo están provocando el aumento en los niveles de desigualdad, sino la aparición de un nuevo fenómeno social, la exclusión de la participación en el ciclo productivo. A diferencia del capitalismo industrial tradicional, que incluía a todos a través de vínculos de explotación y dominación, este nuevo capitalismo tiene una fuerte tendencia expulsora, basada en la ruptura de los vínculos. La exclusión del trabajo es la base de una exclusión social más general o para usar la expresión de Robert Castel —una desafiliación con respecto a las instancias 
sociales más significativas - La exclusión social provoca, desde este punto de vista, una modificación fundamental en la estructura de la sociedad, que estaría pasando de una organización vertical, basada en relaciones sociales de explotación entre los que ocupan posiciones superiores frente a los que ocupan las posiciones inferiores, a una organización horizontal, donde lo importante no es tanto el lugar en la jerarquía sino la distancia con respecto al centro de la sociedad.

El avance de la exclusión tiende, de esta manera, a reemplazar la relación tradicional de explotación. Explotadores y explotados pertenecen a la misma esfera económica y social, ya que los explotados son necesarios para mantener el sistema. La toma de conciencia de la explotación puede provocar -como lo muestra la historia del capitalismo - una reacción de movilización colectiva y de conflicto organizado a través de las instituciones representativas de los explotados. La exclusión, en cambio, no implica relación sino divorcio. La toma de conciencia de la exclusión no genera una reacción organizada de movilización. En la exclusión no hay grupo contestatario, ni objeto preciso de reivindicación, ni instrumentos concretos para imponerla. Siguiendo nuevamente a Castel, mientras que la explotación es un conflicto, la exclusión es una ruptura.

Pero la enorme complejidad de estos procesos se advierte cuando observarnos también qué pasa en la esfera de los incluidos, particularmente de aquéllos que trabajan intensivamente con las nuevas tecnologías. En este ámbito de la organización del trabajo, uno de los fenómenos más importantes es que la pirámide jerárquica tradicional de organización del trabajo está desapareciendo. En la organización del trabajo basada en la utilización intensiva de conocimientos, se tienden a reemplazar las tradicionales pirámides de relaciones de autoridad, por redes de relaciones cooperativas. En este esquema, todas las fases del proceso productivo son importantes y el personal, en cualquier nivel de jerarquía que se ubique, desempeña un papel crucial. El concepto de "calidad total" , que orienta las transformaciones en los actuales modelos de gestión, supone una relación mucho más igualitario que en el pasado entre los que se incorporan a las unidades productivas. Este nuevo modelo de organización del trabajo exige altos niveles de calidad en todas las fases del proceso productivo. La inteligencia no puede estar concentrada en la cúpula de la pirámide sino que debe estar homogéneamente distribuida en toda la red del proceso productivo. El ejemplo clásico que se utiliza para describir esta situación es el comportamiento basado en la hipótesis del "error O' C Como se sabe, esta hipótesis fue elaborada a partir del accidente de la nave espacial Challenger, donde todo el esfuerzo y la inversión realizados para ese proyecto se perdieron por una simple falla en una conexión secundaria. El análisis de este ejemplo permite apreciar que, en el marco de las actuales tecnologías de producción, el menor disfuncionamiento de una de las partes amenaza la producción en su conjunto. En consecuencia, los niveles de calidad y de calificación de los trabajadores que se desempeñan en un mismo proceso productivo deben ser semejantes. Esta mayor igualdad entre los trabajadores del sector tecnológicamente más moderno de la economía exacerba la tendencia a que los mejores tienden a agruparse con los mejores, y los mediocres con los mediocres. De esta forma, cada unidad de producción se transforma en un sub-conjunto homogéneo de un proceso productivo mucho más amplio.

Pero la mayor igualdad entre los incluidos, implica una separación mucho más profunda con respecto a los excluidos. David Cohen, en su reciente libro sobre la riqueza en el mundo, pudo sostener que — al contrario de las hipótesis optimistas de Toffler- las 
economías intensivas en conocimientos y productoras de ideas son más in equitativas que las economías intensivas en personal y que fabrican objetos.

En este contexto, la segmentación y la desigualdad social cambian de sentido. Mientras en la economía capitalista tradicional, cada segmento social era una categoría y la desigualdad se producía entre grupos sociales, ahora, en cambio, la segmentación se produce dentro de cada grupo social. Los datos presentados por Cohen, por ejemplo, indican que más del $70 \%$ del fenómeno de la desigualdad en los EE.UU. se explica por la diferencia de salarios entre trabajadores jóvenes, entre diplomados o entre trabajadores de la industria. Mientras las desigualdades tradicionales eran "intercategoriales", estas nuevas desigualdades son "intracategoriales". En términos subjetivos, una de las características más importantes de este fenómeno es que resulta mucho más difícil de aceptar, porque pone en crisis la representación que cada uno tiene de sí mismo. Estas nuevas desigualdades provocan, por ello, un sufrimiento mucho más profundo, porque son percibidas como un fenómeno más personal que socio económico y estructural.

Por esta razón, no es casual que el aumento de la desigualdad esté acompañado por la difusión de teorías que tienden a justificar este fenómeno a través de la importancia de los factores genéticos en la explicación de determinados patrones de conducta, de los niveles de desarrollo cognitivo personal y de la ubicación en la estructura social.

Una de las versiones más difundidas de este neo-darwinismo social la constituye el libro de Richard J. Hermstein y Charles Murray sobre la inteligencia y la estructura de clases sociales en los EE.UU., basado en el supuesto según el cual la habilidad cognitiva será la variable decisiva en la estructura social que se está conformando para el nuevo siglo y que dicha habilidad es fundamentalmente hereditaria. Algunos científicos sociales han asumido plenamente este enfoque que, paradójicamente, anula cualquier posibilidad de análisis social de las conductas humanas, Francis Fukuyama, por ejemplo, ha sostenido en uno de sus más recientes ensayos, que los comportamientos sociales se explican por factores genéticos y aquellos comportamientos para los cuales aún no se ha descubierto su relación con los genes, existen buenas razones para esperar que dicha relación será descubierta en las próximas décadas. Para Fukujama, como para otros ensayistas de esta misma corriente, fenómenos como la criminalidad, la drogadicción, el alcoholismo, la promiscuidad, las separaciones, los divorcios y otras "conductas desviadas", estarían explicados por factores genético-hereditarios y, por tanto, difícilmente modificables a través de políticas sociales.

La justificación de la desigualdad a partir de los resultados de la investigación genética es uno de los principales ejemplos acerca del papel que realizará el conocimiento en la determinación de la estructura social. La información genética permitirá predecir trayectorias de vida con mucha más precisión que en el pasado y la utilización de esta información tiene potencialidades enormes sobre todo el sistema de relaciones sociales. Jeremy Ritkin, el autor de El fin del trabajo, ha dedicado su último libro a la revolución bío - tecnológica, donde muestra ejemplos de cómo no sólo las compañías de seguros pueden usar la información genética para definir qué tipo de tratamiento brindarán a los asegurados, sino que los empleadores pueden también aplicar estos resultados en sus políticas de reclutamiento de personal y las escuelas en el reclutamiento de sus alumnos. Se abre así la posibilidad de una sociedad organizada en nuevas y más virulentas formas de discriminación, basadas en el perfil genético de cada uno. 
Obviamente, estos escenarios futuros no son inevitables. Ya aprendimos que el futuro nunca está escrito y que el conflicto entre el desarrollo de opciones basadas en el principio de justicia y de democracia y las opciones autoritarias e injustas socialmente seguirá siendo una constante del desarrollo de la sociedad. Pero este análisis nos muestra que luchar por la justicia, particularmente por la justicia social, no tiene y no tendrá en el futuro, los mismos componentes ni las mismas formas que en el pasado.

La centralidad del conocimiento en la sociedad y la disponibilidad de información sobre cada uno, disminuye la posibilidad de continuar administrando justicia según el principio del "velo de la ignorancia", presentado por John Rawls en su teoría de la justicia. La justicia, en el estado-providencia y en todas las formas tradicionales de solidaridad, funcionaba sobre la base de este velo de ignorancia que no indaga sobre las particularidades de cada individuo. La ignorancia, en este sentido, contribuye a la cohesión social, mientras que el conocimiento y las informaciones sobre las particularidades de cada individuo ponen en marcha mecanismos de desolidarización, de ruptura de la cohesión y de debilitamiento del papel de la socialización.

Frente a estas tendencias y como respuesta a las ideologías neo-conservadoras y neodarwinianas, que justifican la desigualdad social, se está gestando un nuevo pensamiento democrático, basado en la idea según la cual eliminar la desigualdad puede y no debe ser contradictoria con el respeto a la diversidad y a la identidad personal de cada uno. De acuerdo a estos postulados, es necesario mantener la vigencia de los valores de justicia y de solidaridad como elementos básicos para garantizar el carácter sostenido del desarrollo social. Pero esos principios de justicia y equidad ya no pueden ser aplicados de la misma manera que en el pasado. La justicia, por ejemplo, no puede estar basada en la idea de tratar a todos de la misma manera. La justicia, particularmente la justicia social, debe perder el velo que cubre sus ojos y que le impide ver a quien se dirige y tratarlo de la manera más adecuada a su situación. La mayor disponibilidad de información puede, desde este punto de vista, ser la base de estrategias de acción social más eficaces para el logro de la justicia y no, como lo sugieren los enfoques conservadores, un factor de discriminación.

Pero este nuevo enfoque de la justicia y de la solidaridad social está íntimamente asociado al fortalecimiento de la dimensión política de la sociedad y, en particular, de la democracia. En definitiva, un desarrollo social basado en la idea de justicia, un desarrollo social que tenga sentido a largo plazo, que implique un proyecto de civilización, supone pasar de la actual subordinación de las nuevas tecnologías de producción a la lógica del mercado, a la subordinación a la lógica de la ciudadanía. La inclusión de todos es un proyecto que tiene sentido desde el punto de vista político, desde el punto de vista de un proyecto que, para usar los términos del Informe de la Comisión de la UNESCO presidida por Jacques Delors, nos permita vivir juntos.

La incorporación de la dimensión política en este análisis nos obliga a observar el otro gran fenómeno que vivimos actualmente: la crisis del Estado-Nación y la expansión de lo que algunos llaman la globalización o la mundialización de las relaciones sociales.

Desde el punto de vista económico, la globalización no significa sólo que los capitales puedan moverse rápida y libremente por todo el planeta. El fenómeno socialmente más importante es que como las empresas pueden instalarse en cualquier parte del mundo y mantenerse conectadas a través de redes de información, ellas tienden a radicarse allí 
donde los costos son menores. Este fenómeno produce lo que se ha denominado "una espiral descendente de reducción de costos sociales", que tiende a debilitar la capacidad de los estados-nacionales para mantener los niveles tradicionales de beneficios sociales y de bienestar. La globalización económica, en síntesis, reduce la capacidad del estado para definir su política monetaria, su presupuesto, su recaudación de impuestos y la satisfacción de las necesidades sociales de su población.

Al estar basada fundamentalmente en la lógica económica y en la expansión del mercado, la globalización rompe los compromisos locales y las formas habituales de solidaridad y de cohesión con nuestros semejantes. Las élites que actúan a nivel global tienden a comportarse sin compromisos con los destinos de las personas afectadas por las consecuencias de la globalización. La respuesta a este comportamiento por parte de los que quedan excluidos de la globalización es el refugio en la identidad local donde la cohesión del grupo se apoya en el rechazo a los "externos".

En este sentido, numerosos diagnósticos de la sociedad actual muestran que la ruptura de los vínculos tradicionales de solidaridad provocada por el proceso de globalización ha generado nuevas formas de exclusión, de soledad y de marginalidad. Las formas de asociación y de expresión de algunos de estos sectores excluidos tienden a apoyarse en valores de intolerancia, de discriminación y de exacerbación de los particularismos. Mientras en la cúpula, las elites que participan de la economía supranacional plantean el riesgo de que su desapego a la nación estimule un individualismo a-social, basado en la falta total de solidaridad, en la base se aprecian fenómenos regresivos de rechazo al diferente, de xenofobia y de cohesión autoritaria.

Manuel Castells, en ese enorme esfuerzo de análisis sobre la Era de la Información donde, en tres volúmenes, ofrece un panorama exhaustivo de la economía, la sociedad y la cultura contemporáneas, explica con claridad este proceso, que da lugar a la aparición del fundamentalismo y de los estados fundamentalistas. De acuerdo a su análisis, el estado-nación, para sobrevivir a su crisis de legitimidad, cede poder y recursos a los gobiernos locales y regionales. En este proceso, pierde capacidad para igualar los intereses diferentes y representar el "interés general". Este proceso deslegitima aún más al Estado, particularmente frente a las minorías discriminadas, que buscan protección en las comunidades locales o en otro tipo de estructuras. Según Castells "...lo que comenzó como un proceso de relegitimación del estado, mediante el paso del poder nacional al local, puede acabar profundizando la crisis de legitimación del estado-nación y la tribalización de la sociedad en comunidades construidas en torno a identidades primarias".

En un contexto de este tipo, la construcción de alternativas democráticas a las tendencias actuales que, tras la apariencia de modernización, suponen un retomo a la barbarie, implica re-valorizar el objetivo de la cohesión social, de la dimensión política de la sociedad y de la socialización de las personas en función de valores que promuevan la solidaridad con el semejante y con el diferente. Pero a diferencia de la sociedad, la política y la cultura en el capitalismo tradicional, la cultura en esta era de la información no podrá ser impuesta desde afuera del sujeto por instituciones de socialización sino que debe ser construida por cada uno.

La opción sarmientina de "civilización o barbarie" vuelve así a cobrar plena vigencia. Pero la diferencia entre el final del siglo XIX y el final del siglo XX es que el objetivo de 
fortalecer la cohesión social no puede ser encarado por la imposición de un único modelo ideológico, sino sobre la base del desarrollo del sujeto a través de la formación de sus competencias para construir su propia identidad, uno de cuyos rasgos debe ser la capacidad de aceptar la existencia del "otro", del diferente. La educación, por eso, vuelve a estar en el centro de las estrategias de acción social y política.

Dicho en otras palabras, en el marco de las nuevas configuraciones sociales, las instancias a través de las cuales se producen y se distribuyen el conocimiento y los valores culturales -o sea, las instituciones educativas, los educadores y los intelectuales en genera- ocuparán un lugar central en los conflictos a través de los cuales se definirán las orientaciones de estos procesos sociales.

Si bien no es posible ni en los límites de esta conferencia ni tampoco en los límites de mis capacidades de análisis, hacer una presentación exhaustiva de los nuevos desafíos de la educación, quisiera plantear al menos algunos puntos que me parecen fundamentales.

En primer lugar, este análisis pone en evidencia la importancia crucial que adquiere hoy la definición de políticas educativas que garanticen a todos una educación de muy buena calidad. Tener acceso a una educación de este tipo se ha convertido en la condición necesaria de cualquier estrategia de cohesión social, de participación política, de ingreso al mercado de trabajo y de desarrollo de las competencias básicas que permitan a cada uno la construcción de sus opciones de vida. Una política de este tipo requiere numerosos componentes, pero en el contexto de lo que hemos venido analizando, hay un factor específico que adquiere hoy una renovada importancia: frente a los avances ideológicos de los que quieren mostrar que la capacidad de aprendizaje de las personas está asociada a factores genéticos, será preciso desarrollar con más fuerza que nunca la confianza en la capacidad de aprendizaje de todas las personas, particularmente de aquéllas que nacen en contextos de pobreza y precariedad social.

Desde este punto de vista, es fundamental la prioridad a la democratización del acceso a los circuitos en los cuales se produce y se distribuye el conocimiento socialmente más significativo. La privatización de estos circuitos y su apropiación por un grupo reducido de la población daría lugar a una especie de neo-despotismo ilustrado, incompatible con formas políticas democráticas de participación y control social. Si en el pasado el sistema podía organizarse en niveles que se correspondían con determinadas categorías sociales y de complejidad en la organización del conocimiento, en el futuro la democratización del acceso a los niveles superiores de análisis de realidades y fenómenos complejos debe ser universal. Este acceso universal a la comprensión de fenómenos complejos constituye la condición necesaria para evitar la ruptura de la cohesión social y los escenarios catastrofistas que potencialmente están presentes en las tendencias sociales actuales. Pero el acceso a la comprensión de fenómenos complejos no puede estar asociado a un determinado nivel del sistema y, mucho menos, a sus niveles superiores. La formación básica y universal deberá ser capaz de dotar al conjunto de los ciudadanos de los instrumentos y de las competencias cognitivas necesarias para un desempeño ciudadano activo.

En segundo lugar, es preciso mencionar el cambio en el papel de la educación frente a la movilidad social. En el capitalismo tradicional, la educación estaba directamente asociada a las posibilidades de movilidad social. Ascender en la jerarquía del sistema 
educativo significaba acceder a niveles más complejos del conocimiento y a posiciones más altas en la estructura ocupacional, pero en la medida que la estructura ocupacional de tipo piramidal tiende a perder importancia y se expanden las redes como modelo de organización de las instituciones, disminuye la importancia de la movilidad social vertical y aumentan, en cambio, las posibilidades y exigencias de movilidad horizontal. La educación también modifica su papel ya que, por un lado, será la variable más importante que permitirá entrar o quedar afuera del círculo donde se definen y realizan las actividades socialmente más significativas y, por el otro, será necesario educarse a lo largo de toda la vida para poder adaptarse a los requerimientos cambiantes del desempeño social y productivo. En el futuro, paradójicamente, será necesaria una movilidad muy intensa para mantenerse en la misma posición.

Este fenómeno tiene consecuencias muy importantes sobre el comportamiento de la demanda educativa. Asumir que debemos educarnos a lo largo de toda la vida, que ningún aprendizaje es definitivo y que el acceso al conocimiento no garantiza ascenso social, modifica profundamente la representación social tradicional sobre la educación. Explicar este nuevo sentido de la educación y transformarlo en representación social requerirá no sólo tiempo sino esfuerzos explícitos que ayuden a la población a elaborar demandas educativas más calificadas y, al mismo tiempo, ayuden a comprender el sentido de las transformaciones educativas.

En tercer lugar, es preciso considerar la educación desde el punto de vista del proceso de socialización. Al respecto, ya no es posible pensar, como en el pasado, que las regulaciones vendrán exclusiva o fundamentalmente de instituciones como el Estado, la Iglesia o la familia. Tampoco es posible pensar que habrá una regulación espontánea basada en los mecanismos del mercado, que asegure la cohesión y la equidad necesarias para el desarrollo social sustentable. Las formas tradicionales de solidaridad están perdiendo importancia. Numerosos testimonios indican la aparición y el riesgo de expansión rápida de una sociedad atomizada, donde el individuo aislado estaría frente a una colectividad anónima. Están apareciendo nuevas formas de solidaridad y asociación: círculos de vecinos, "tribus" urbanas, bandas juveniles, etc. Pero las solidaridades generadas por estas nuevas formas de agrupamiento no están asociadas a movimientos integradores. La desaparición de las formas tradicionales de pertenencia obligada, provoca la aparición de una nueva obligación, la de generar uno mismo su forma de inserción social.

Un ejemplo claro de esta transformación es el caso de la familia. La familia mantiene su importancia, pero ya no es la familia fija y estable de antes. La trayectoria familiar de una persona puede atravesar fases diferentes: pareja estable, familia mono parental, unión libre, etc. Los parientes se transforman en una combinación de lazos electivos y de sangre. Lo mismo sucede con el resto de los círculos (amigos, colegas, etc.). En este contexto, se estaría configurando un tipo de sociedad donde hay el riesgo de la existencia de formas paralelas, duales, de pertenencia social. Por un lado, ámbitos donde predominarán las relaciones elegidas y especializadas y, por el otro, ámbitos donde volverán a establecerse solidaridades impuestas por factores adscriptivos. Más allá del análisis de cada una de estas posibilidades, lo cierto es que el papel y las formas de solidaridad serán el tema central en la discusión acerca de las alternativas de desarrollo social en el futuro. 
La formación del sentido de solidaridad está íntimamente asociada a la formación del sentido de pertenencia. Al respecto, el desafío educativo implica desarrollar la capacidad de construir una identidad compleja, una identidad que contenga la pertenencia a múltiples ámbitos: local, nacional e internacional, político, religioso, artístico, económico, familiar, etc. Lo propio de la ciudadanía moderna es, precisamente, la pluralidad de ámbitos de desempeño y la construcción de la identidad a partir precisamente de esta pluralidad y no de un solo eje dominante y excluyente o plural de pertenencia, que en términos educativos, el desarrollo de este sentido combine la adhesión y la solidaridad local con la apertura a las diferencias, implica introducir masivamente en las instituciones escolares la posibilidad de realizar experiencias que fortalezcan este tipo de formación. Al respecto, todos los diagnósticos indican la existencia de un significativo déficit de experiencias democráticas y pluralistas en la sociedad. La escuela es un ámbito privilegiado para el desarrollo de experiencias de este tipo, que puedan ser organizadas educativamente.

Postular la necesidad de desarrollar este conjunto de competencias y capacidades es necesario pero no suficiente. El desafío para los educadores consiste, además, en definir los diseños institucionales más apropiados y elaborar las herramientas técnicas y metodológicas más eficaces para que estos objetivos superen la fase puramente retórica y se transformen en metas concretas de aprendizaje. Desde el punto de vista institucional, es necesario discutir qué tipo de escuela y qué articulaciones entre ellas y la sociedad son las más apropiadas para estos desafíos. La escuela tradicional ha estado particularmente cerrada al contacto con otras instituciones y con otros actores sociales. Si bien este desafío podía ser el más apropiado en el momento de construcción de los estadosnacionales, cuando la escuela aparecía como la institución que debía superar los particularismos, actualmente ya no puede mantenerse aislada, ignorando las transformaciones que se han producido en el ámbito de la familia, de la empresa y de los medios de comunicación.

En síntesis, es preciso romper el aislamiento institucional de la escuela, abriéndola a los requerimientos de la sociedad y redefiniendo sus pactos con los otros agentes socializadores, particularmente la familia y los medios de comunicación. Pero, ¿cuál debería ser el papel específico de la escuela? En el contexto del análisis que efectuamos hasta aquí, parece necesario enfatizar la idea que la escuela debe asumir una parte significativa de la formación en los aspectos "duros" de la socialización. Esto no significa reivindicar la rigidez, la memoria, la autoridad, etc., sino aceptar que su tarea es llevar a cabo en forma consciente y sistemática, la construcción de las bases de la personalidad de las nuevas generaciones.

En un mundo donde la información y los conocimientos se acumulan y circulan a través de medios tecnológicos cada vez más sofisticados y poderosos, el papel de la escuela debe ser definido por su capacidad para preparar para el uso consciente, crítico, activo, de los aparatos que acumulan la información y el conocimiento. En este sentido, parecería que una de las pistas más prometedoras de trabajo para la escuela es la que tiene que ver justamente con su relación con la convivialidad, con las relaciones cara a cara, con la posibilidad de ofrecer un diálogo directo, un intercambio con personas reales donde los instrumentos técnicos sean lo que son, instrumentos y no fines en sí mismos. El clima de las instituciones escolares, diferenciadas según proyectos pedagógicos y dotadas de significativos niveles de autonomía para poder conectarse con el medio, constituye una variable central para el desarrollo de un proceso de socialización eficaz. 
Pero así como el diseño institucional tradicional no puede ser mantenido en las actuales circunstancias históricas, también es preciso advertir que un diseño institucional basado solamente en la autonomía de las escuelas puede aumentar los riesgos de atomización y fragmentación social y cultural. Desde este punto de vista, la autonomía debe ser un estímulo para la vinculación y no para el aislamiento. La idea de red constituye una forma fértil para estimular conexiones entre las instituciones escolares que superen el formalismo tradicional y permitan intercambios reales, tanto a nivel local como nacional e internacional.

Quisiera cerrar esta exposición con una pregunta y una preocupación que seguramente muchos de ustedes compartirán: ¿cuánto y cómo este debate, estas perspectivas, estos conflictos y desafíos son pertinentes para los países de América Latina de hoy?

Estoy persuadido que nuestros países no están o, mejor dicho, no deberían estar ajenos ni ausentes de este debate. Lo peor que nos puede pasar es quedar atados a la lógica de las visiones simplificadoras de corto plazo de los que quieren reducir esta cuestión a un problema de mercado, de equilibrio contable o de meros procedimientos de gestión administrativa. Estamos ante el desafío de articular los esfuerzos de la transformación educativa con una transformación social con visión civilizatoria. Frente a la sacralización de la urgencia y del corto plazo, parece imprescindible asumir socialmente que el largo plazo, que la definición del sentido de hacia donde queremos ir, también es urgente. 